\title{
Free Website Materials
}

by Jeremy Adams and Siong $N g$
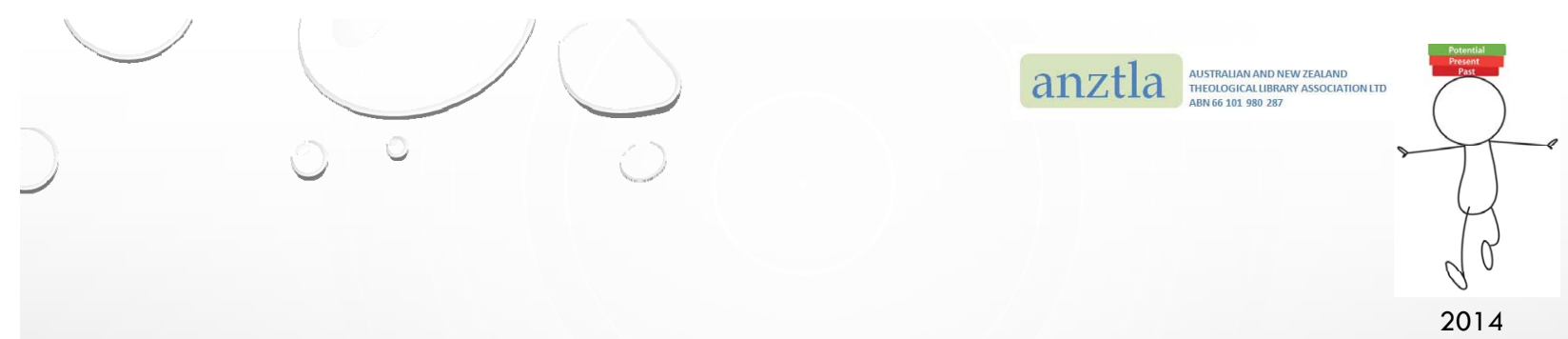

FREEBIES FOR YOUR WEBSITE? 

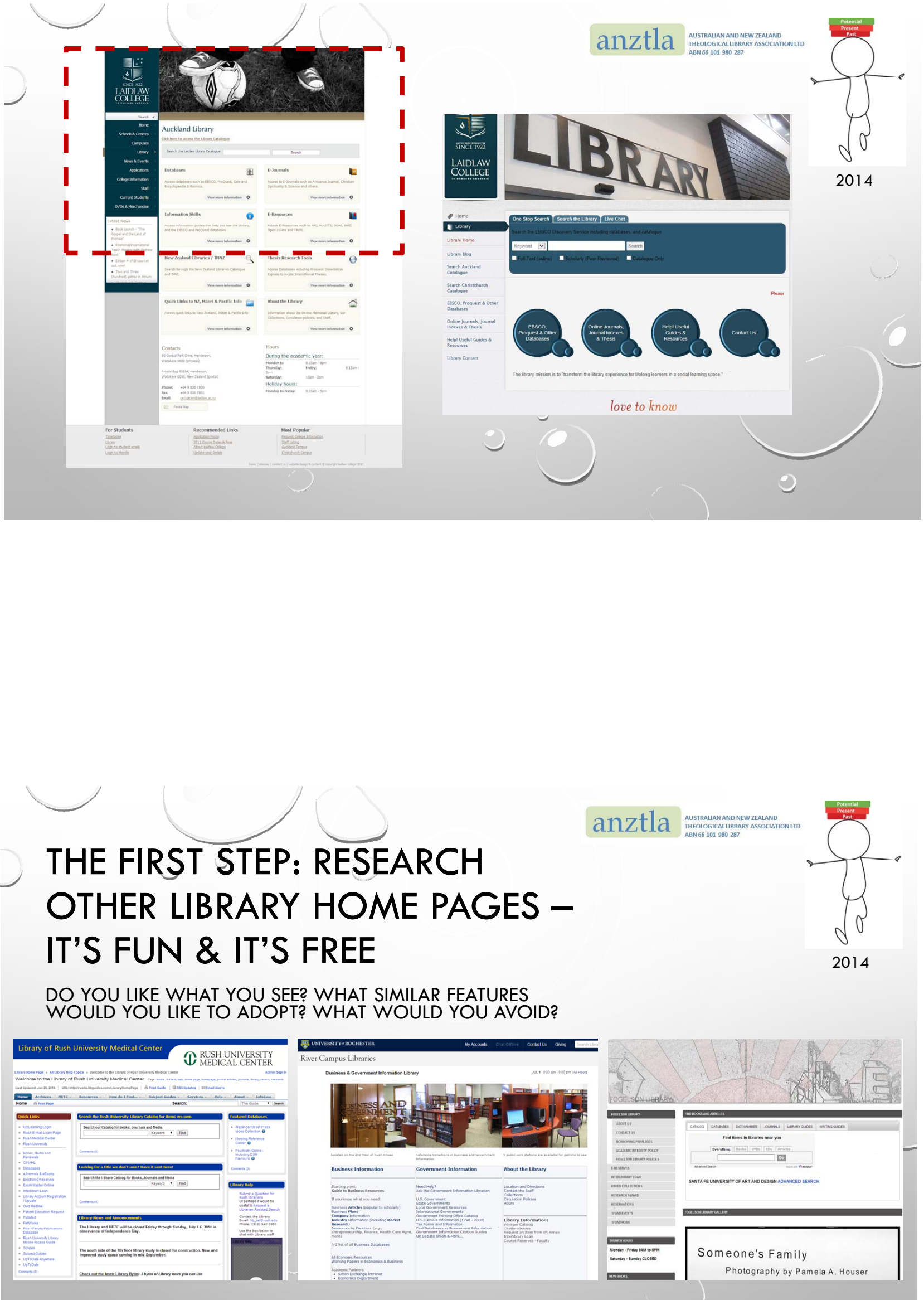


\section{Read!}

\section{anztla}

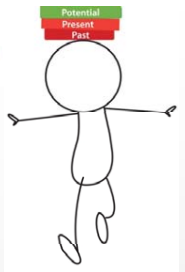

Articles:

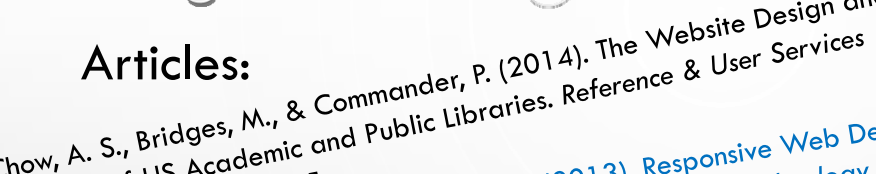

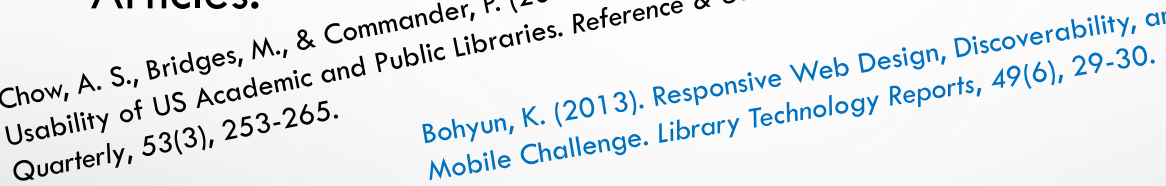

Darby, A., \& Gilmour, R. (2009). Tutorial: Adding Delicious Data to Your Library Website. Information Technology \& Libraries,

Blogs: 28(2), 100-103.

http://www.slideshare.net/thewikiman/marketing-libraries-ina-web-2-world
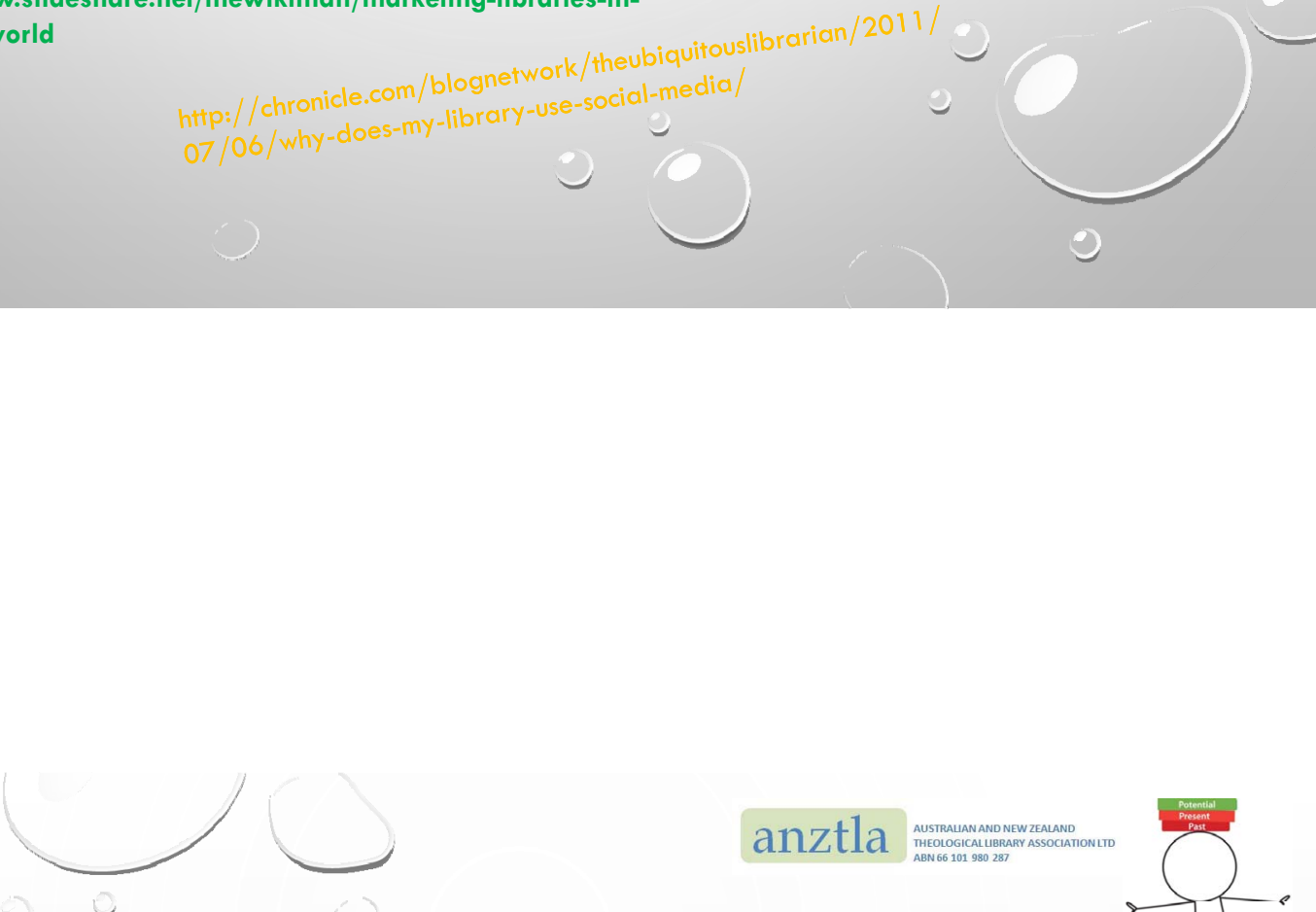

\section{anztla}
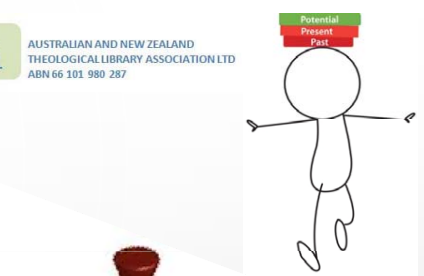

GET YOURSELF OUT THERE!
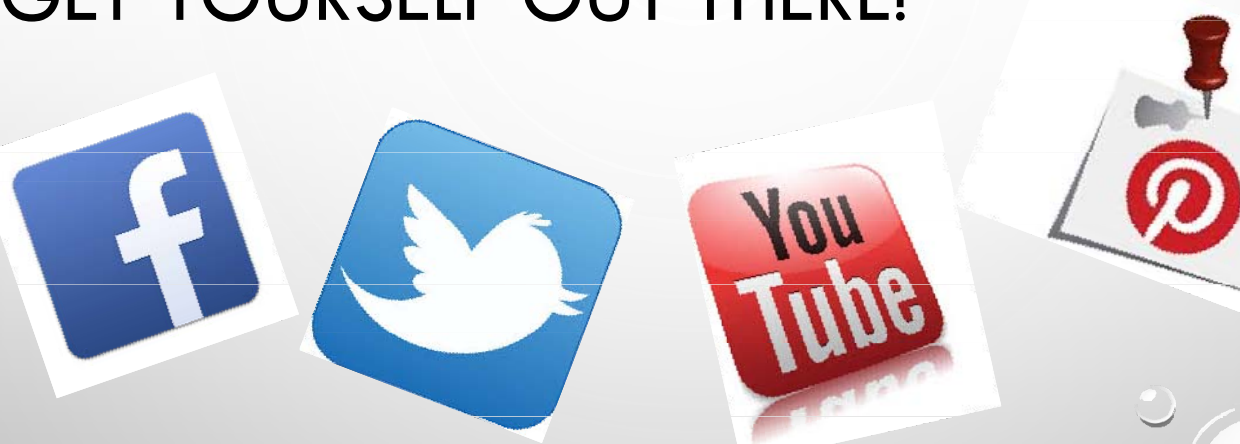

2014 
AND TALKING ABOUT SOCIAL MEDIA...
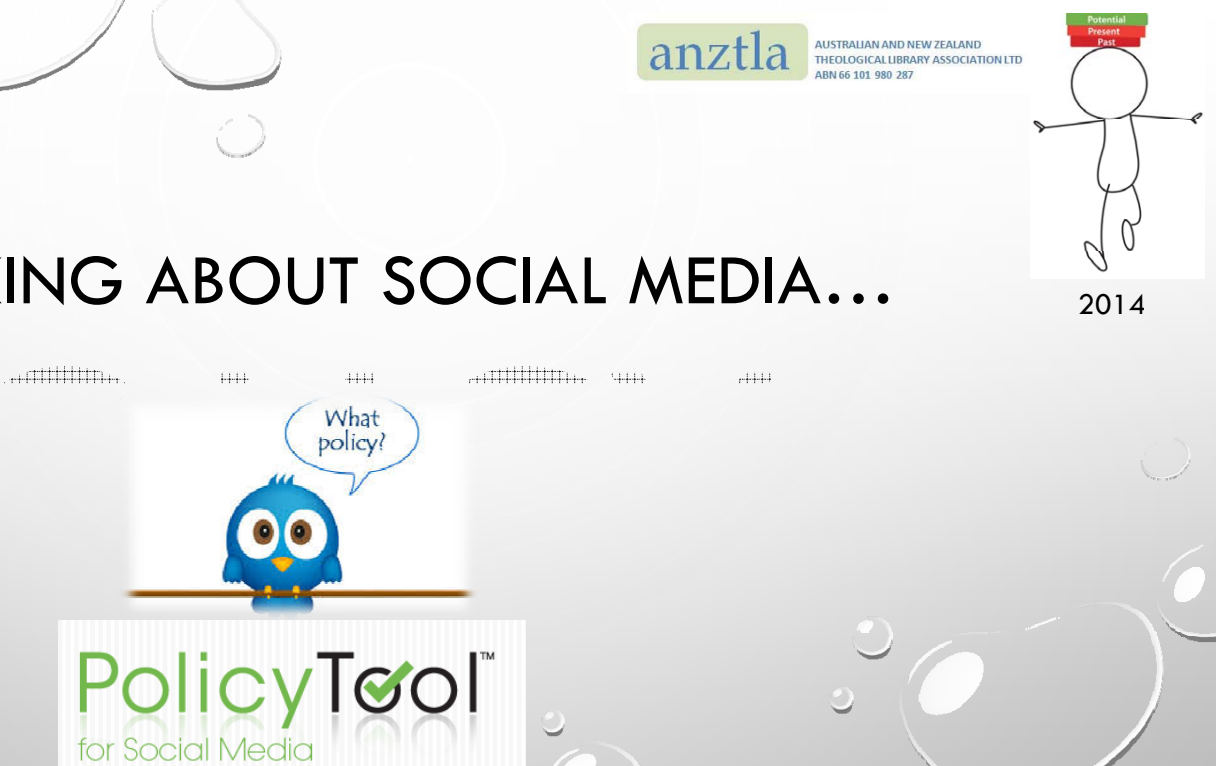

http://socialmedia.policytool.net/index.php/welcome/wizard

WiX HTTP://WWW.WIX.COM

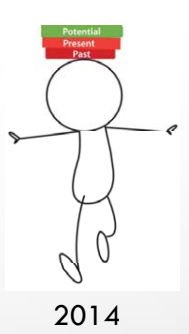

(yola HTTPS://WWW.YOLA.COM

\section{बquackff ${ }^{\text {.oom }}$ HTTP://WWW.QUACKIT.COM}




\section{Icon finder}

\section{https://www.iconfinder.com/}

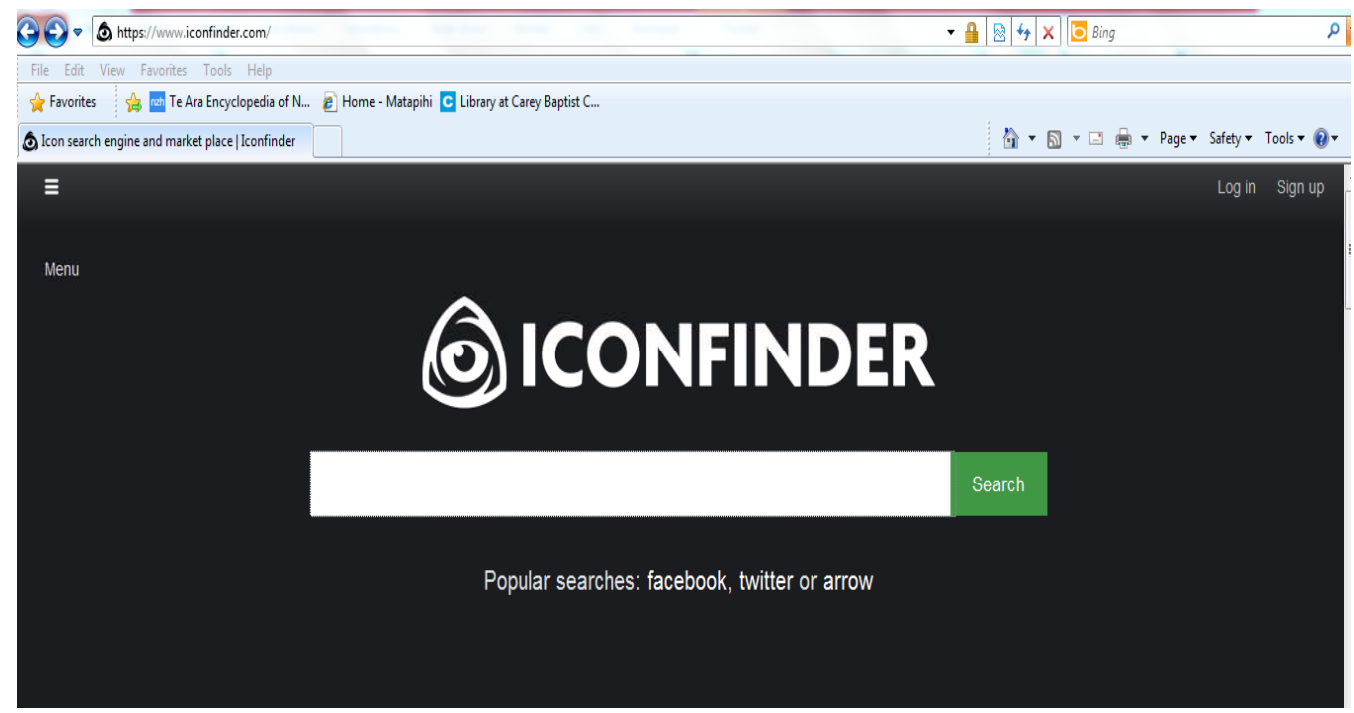

\section{Microsoft free clip art}

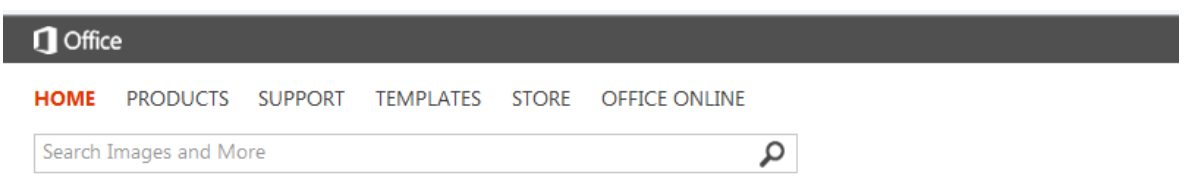

Back to school with Office Clip Art and Media

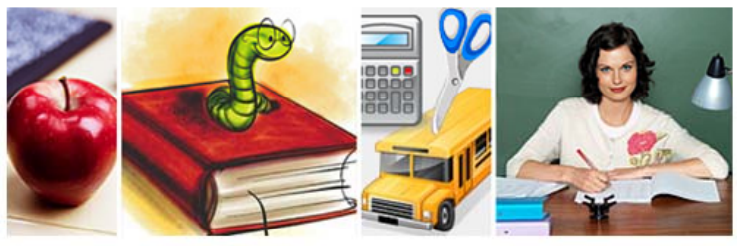

Teachers, students, parents: We want to help make your job easier and more fun. If you're looking for free images, animations, or sounds, look no further: we have over 150,000 clips for you to pick from. 
E PRODUCTS SUPPORT TEMPLATES STORE OFFICE ONLLNE
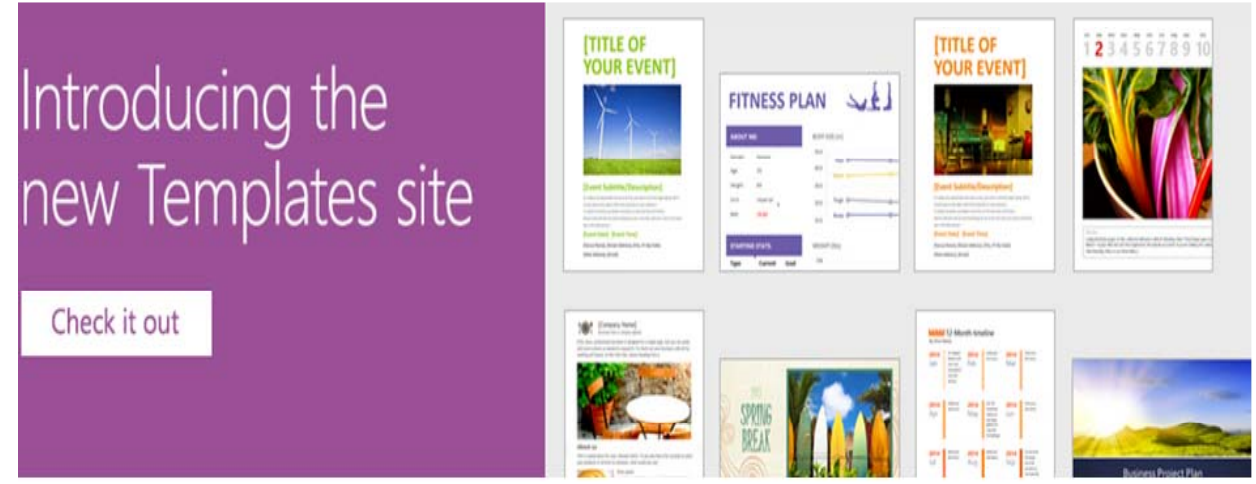

\section{Scroll down}

All categories for online templates

\begin{tabular}{llll} 
Agendas & Certificates & Letters & Planners \\
Blank & Diagrams & Lists & Posters \\
\hline Brochures & Education & Maps & Resumes \\
\hline Budgets & Fax Covers & Memos & Schedules \\
Business & Financial Management & Minutes & Themes \\
Calendars & Flyers & Nature & Timelines \\
Cards & Invoices & Photo Albums & Trackers
\end{tabular}




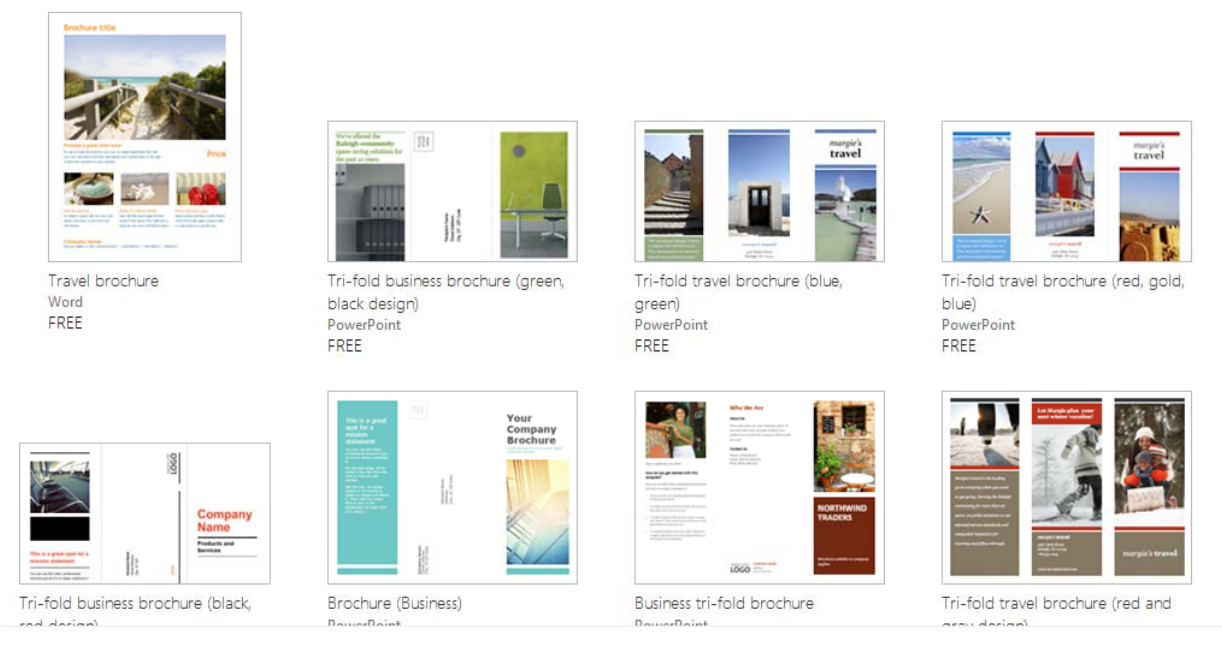

\section{CONTENTdm}

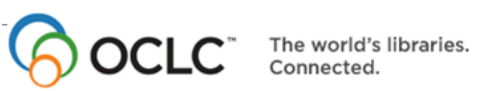

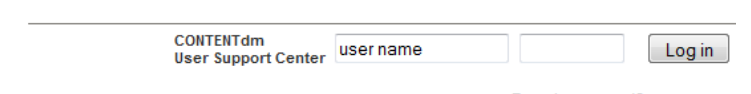

Forgot password?

About $0 \mathrm{Cl} \mathrm{C}$ News and Fvents Contactus

\begin{tabular}{l|l|l|l|l|l|} 
CONTENTdm Overview & CONTENTdm in Action & Free Evaluation & Ordering & User Support Center & \\
\hline
\end{tabular}

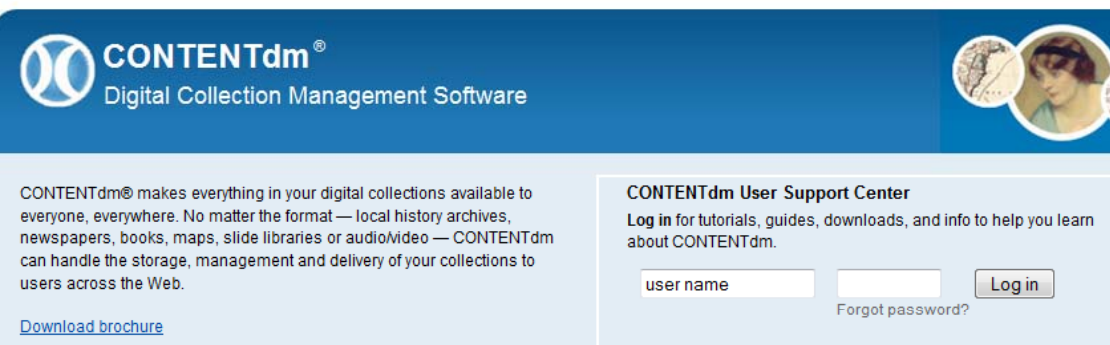

Reaister: First-time users must register

Learn more at www. oclc.org $\gg$ 


\section{Tellagami}
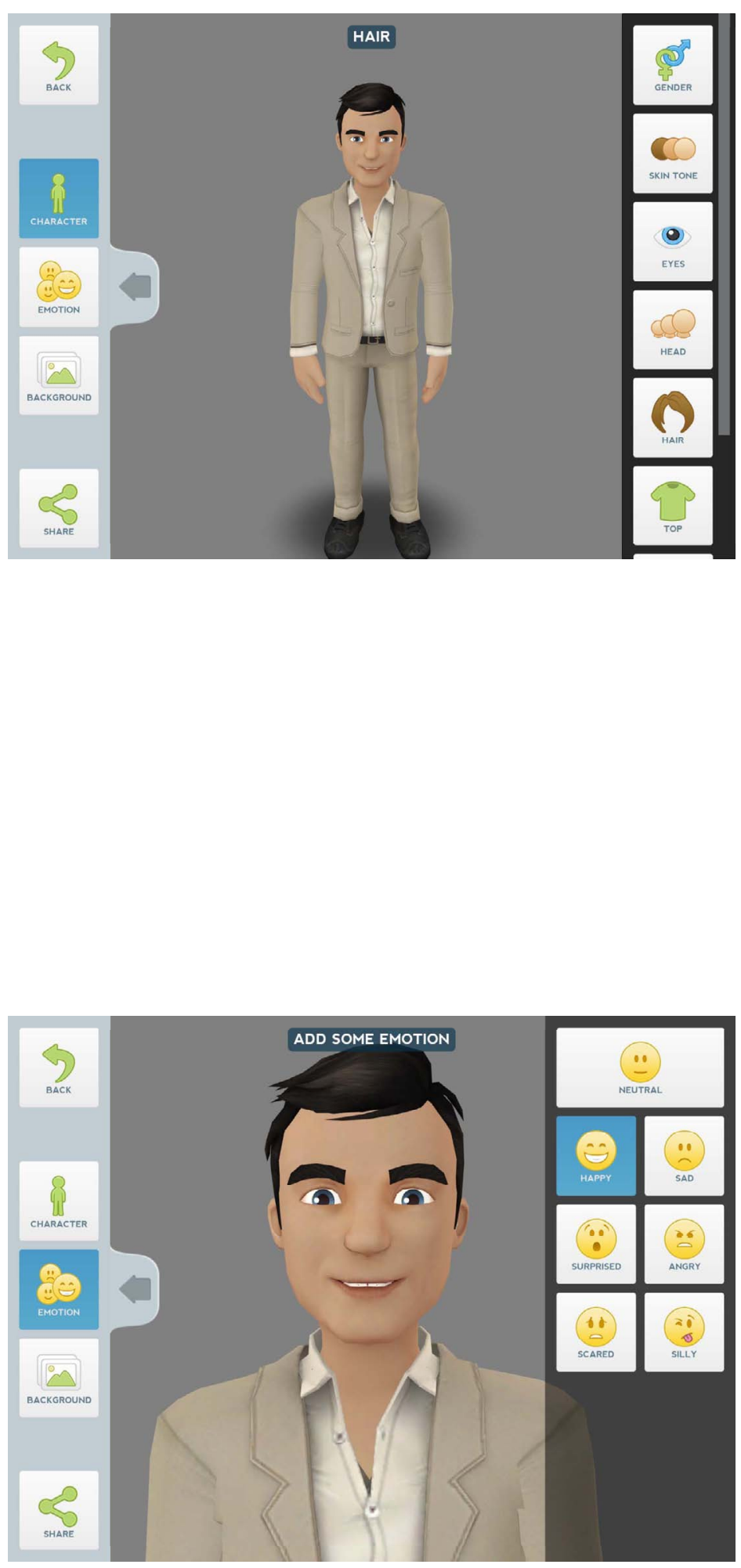

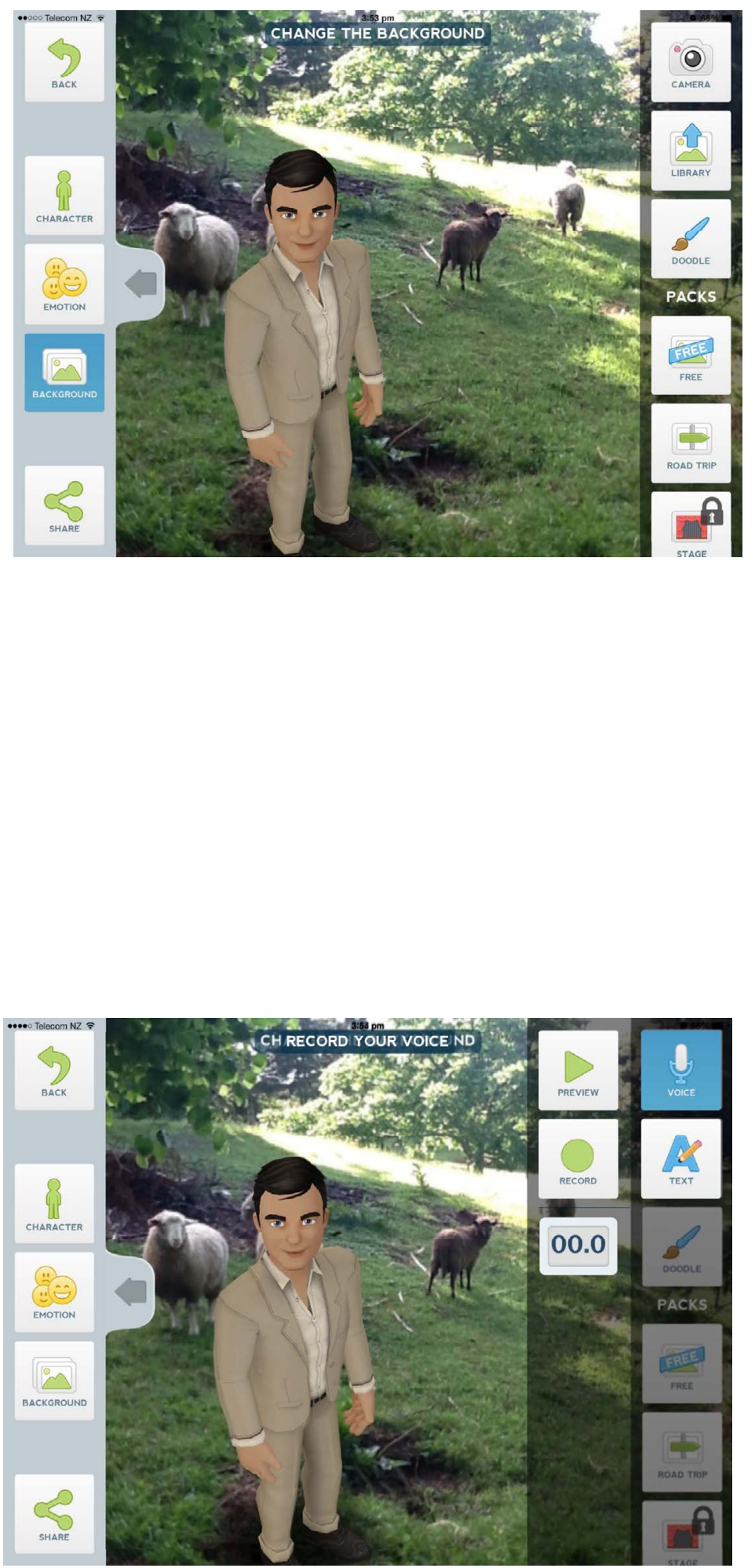


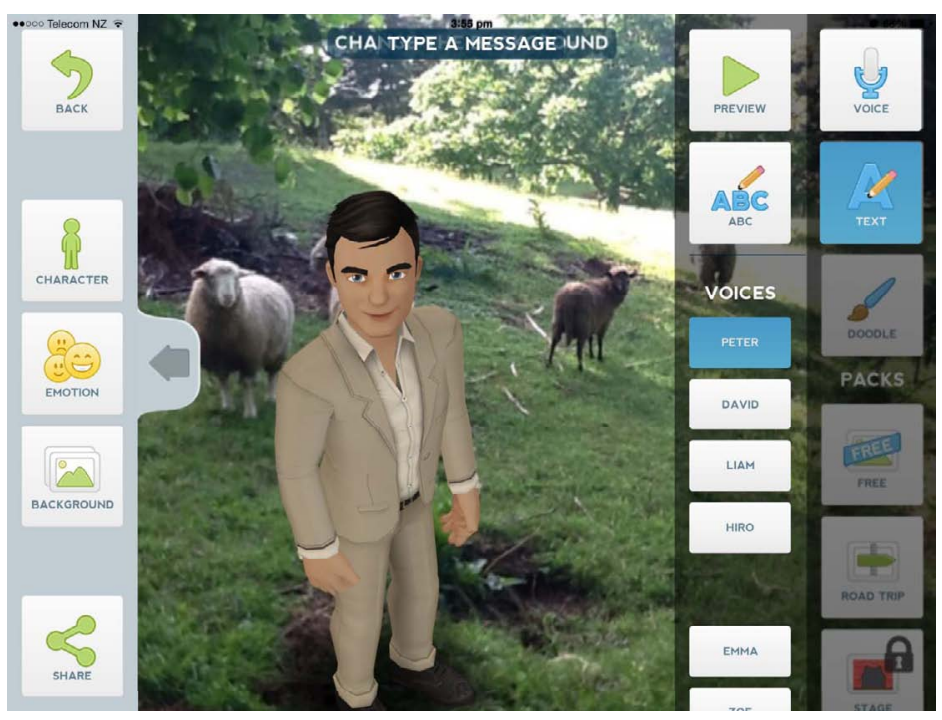

